\title{
Peripheral nervous system effects in the PS19 tau transgenic mouse model of tauopathy
}

\author{
Jesús Merchán-Rubira ${ }^{a}$, Álvaro Sebastián-Serrano ${ }^{\mathrm{b}, \mathrm{c}, 1}$, Miguel Díaz-Hernández ${ }^{\mathrm{c}}$, Jesús Avila ${ }^{\mathrm{a}, \mathrm{b}}$, \\ Félix Hernández ${ }^{\mathrm{a}, \mathrm{b}, *}$ \\ ${ }^{a}$ Centro de Biología Molecular Severo Ochoa (CSIC-UAM), Madrid, Spain \\ ${ }^{\mathrm{b}}$ CIBERNED, Centro de Investigación Biomédica en Red de Enfermedades Neurodegenerativas, Spain \\ ${ }^{\mathrm{c}}$ Departamento de Bioquímica y Biología Molecular, Facultad de Veterinaria, Universidad Complutense de Madrid, 28040, Madrid, Spain
}

\section{A R T I C L E I N F O}

\section{Keywords:}

Sciatic nerve

Neurodegeneration

Tauopathies

\begin{abstract}
A B S T R A C T
It is well known that transgenic mice overexpressing human tau protein with P301S mutation driven by the mouse prion protein promoter show clasping and limb retraction, hunched back and paralysis, followed by inability to feed that results in death around 12 months of age. To understand these motor deficits, we have carried out rotarod tests on PS19 line and demonstrated how they worsened during aging. Then, we have analyzed if these phenotypic characteristics correlate with sciatic nerve degeneration. We first demonstrated by western blot and immunohistochemistry that the sciatic nerve expresses the transgenic tau protein; then, electron microscopy studies showed alterations in myelin, mainly a detachment of myelin lamellae at SchmidtLanterman clefts. Similar motor deficits and myelin alterations have been previously reported in tau knockout and overexpressing transgenic mice; taking into account that PS19 model is widely used to study tauopathies, we suggest that analyzing the expression of transgenic tau protein and myelin abnormalities in the sciatic nerve should be considered when studying some features as motor performance or survival.
\end{abstract}

\section{Introduction}

Tau is a cytoskeletal protein that is expressed mainly in neurons and is involved in several cellular processes such as microtubule stabilization, axonal maintenance, and transport. Altered tau metabolism is related to different tauopathies being the most important Alzheimer disease (AD), where aberrant hyperphosphorylated and aggregated tau is found in the central nervous system (CNS) [8]. Alternative splicing of exons 2, 3, and 10 of microtubule-associated protein tau (MAPT) gene results in the appearance of six tau isoforms in the CNS. Exons 2 and 3 can be alternatively spliced; exon 2 can appear alone (isoforms called $1 \mathrm{~N}$ ), but exon 3 never appears independently of exon 2 (isoforms called $2 \mathrm{~N}$ when both present) [2]. Because exon 10 encodes for one of the regions involved in the binding of tau to microtubules, alternative splicing of exon 10 produces tau isoforms with either three (3R tau, without exon 10) or four (4R tau, with exon 10) microtubule-binding regions. In humans, tau is expressed as both $3 \mathrm{R}$ and $4 \mathrm{R}$ isoforms; however, only $4 \mathrm{R}$ tau is expressed in adult mice, although $3 \mathrm{R}$ isoform can be found in adult newborn neurons generated in the dentate gyrus and subventricular zone [13]. In the peripheral nervous system (PNS), there is a high-molecular-weight tau isoform expressing the exon 4A, which yields a protein known as "big tau" with an approximate size of $100 \mathrm{kDa}[4,6]$.

MAPT gene mutations have not been found in $\mathrm{AD}$, but they cause instead a different subtype of tauopathies, demonstrating that altered tau metabolism is sufficient for neurodegeneration [8]; many of these mutations affect tau splicing to favor the $4 \mathrm{R}$ isoform, although coding mutations have also been described. Multiple transgenic mouse lines have been generated to overexpress human $4 \mathrm{R}$ tau containing some of these mutations; PS19 is one of the most frequently used [10]. The PS19 model is a transgenic line carrying the human 1N4R MAPT gene with the P301S mutation under the control of the mouse prion protein promoter (Prnp); these mice present an increase of around 5-fold in tau levels with the onset of phospho-tau pathology at around 6 months of age and are characterized by the absence of A $\beta$ plaques [15]. PS19 mice undergo a progressive accumulation of insoluble and highly

\footnotetext{
*Corresponding author at: Centro de Biología Molecular "Severo Ochoa", Consejo Superior de Investigaciones Científicas (CSIC), Universidad Autónoma de Madrid (UAM), 28017 Madrid, Spain.

E-mail address: fhernandez@cbm.csic.es (F. Hernández).

${ }^{1}$ Present address: Instituto de Investigaciones Biomédicas “Alberto Sols”, Consejo Superior de Investigaciones Científicas-Universidad Autónoma de Madrid (CSICUAM), Madrid, Spain.
} 
phosphorylated tau species in structures of the CNS; they develop hindlimb paralysis and then die at around 1 year of age.

Here we have explored in PS19 mice the status of sciatic nerve; our results suggest that at least some problems related to their paralysis can be due to the degeneration of this nerve, likely because transgenic tau can be found in this structure.

\section{Materials and methods}

\subsection{Animals, sacrifice and sciatic nerve extraction}

The PS19 mice were obtained from The Jackson Laboratory: line B6;C3-Tg(Prnp-MAPT*P301S)PS19Vle/J; stock number 008169 [15]. The original $\mathrm{B} 6 \mathrm{C} 3 \mathrm{H} / \mathrm{F} 1$ genetic background was changed to $\mathrm{C} 57 \mathrm{BL} / 6 \mathrm{~J}$ by back-crossing them with C57 animals in our laboratory. All mice were housed at the "Centro de Biología Molecular Severo Ochoa" animal facility; they were kept four per cage with food and water available ad libitum and maintained in a temperature-controlled environment on a 12/12 h light/dark cycle with light onset at 07:00 h. Animal housing and maintenance protocols followed the guidelines of the Council of Europe Convention ETS123, revised as indicated in the Directive 86/609/EEC. Animal experiments were performed under protocols (P15/P16/P18/P22) approved by the Institutional Animal Care and Utilization Committee (Comité de Ética de Experimentación Animal del CBM, CEEA-CBM, Madrid, Spain). Animals were sacrificed by a lethal intraperitoneal injection of sodium pentobarbital $(200 \mathrm{mg} / \mathrm{kg}$ b.w.) and were then perfused intracardially with $0.9 \%$ saline. The sciatic nerve was exposed after holding both hind limbs together in one hand and the tail was gently pulled away in the opposite direction as described previously [3]. Left sciatic nerves were frozen immediately to $-80^{\circ} \mathrm{C}$ for protein analysis, while the right ones were used for both immunohistochemistry and electron microscopy.

\subsection{Western blotting}

Extracts for western blotting analysis were prepared by homogenizing the sample tissue in ice-cold extraction buffer consisting of $50 \mathrm{mM}$ Tris- $\mathrm{HCl}, \mathrm{pH} 7.4,150 \mathrm{mM} \mathrm{NaCl}, 1 \%$ Nonidet P-40, $1 \mathrm{mM}$ sodium orthovanadate, $1 \mathrm{mM}$ EDTA, a protease inhibitor mixture COMPLETE ${ }^{\mathrm{Tm}}$ (Roche), and $1 \mu \mathrm{M}$ okadaic acid. Protein content was determined by the Bradford method. $20 \mu \mathrm{g}$ of total protein were electrophoresed on a $10 \%$ SDS-polyacrylamide gel and transferred to a nitrocellulose membrane (Schleicher \& Schuell). Prior to antibody binding, membranes were blocked with $5 \%$ nonfat dried milk. The following primary antibodies were used: mouse anti-GADPH (1/5000, Abcam), mouse anti-tau TAU-5 (total tau; 1/1000, Merck Millipore), and mouse anti-tau AT8 (phosphotau; 1/100, Thermo Fisher Scientific). The membranes were incubated with the antibodies at $4{ }^{\circ} \mathrm{C}$ overnight. Secondary goat anti-mouse antibody (1/5000, Dako), conjugated with HRP, and ECL detection reagents (Amersham Biosciences) were used for immunodetection.

\subsection{Immunohistochemistry}

The sciatic nerves were fixed overnight at $4{ }^{\circ} \mathrm{C}$ in $4 \%$ paraformaldehyde in $0.1 \mathrm{~N}$ phosphate buffer (PB). After inclusion in $4 \%$ agarose- $10 \%$ sucrose in $0.1 \mathrm{~N} \mathrm{~PB}$, sections with a thickness of $50 \mu \mathrm{m}$ were cut on a Leica VT1000S vibratome. Sections underwent a process of floating immunoprecipitation of 3,3'-diaminobenzidine (DAB) with anti-tau HT7 antibody (human; 1/100, Thermo Fisher Scientific) using VECTASTAIN Elite ABC kit (Vector Laboratories), with chromogen reactions performed with SIGMAFAST DAB (Sigma-Aldrich) for $10 \mathrm{~min}$; manufacturer's protocols were followed. Finally, sections were mounted on glass slides and coverslipped with FluorSave (Calbiochem). Images were captured using an Olympus BX41 microscope with an Olympus ColorView IIIu CCD camera (2576 × 1932 pixels).

\subsection{Transmission electron microscopy (TEM)}

The sciatic nerves were fixed in $4 \%$ paraformaldehyde and $2 \%$ glutaraldehyde in $0.1 \mathrm{~N} \mathrm{~PB}$ for $2 \mathrm{~h}$ at room temperature. Next, they were fixed with $1 \%$ osmium tetroxide in water at $4{ }^{\circ} \mathrm{C}$ for $1 \mathrm{~h}$, dehydrated with ethanol, and embedded in TAAB 812 epoxy resin. Ultrathin sections of $80 \mathrm{~nm}$ were cut with an ultramicrotome Ultracut $\mathrm{E}$ (Leica) and mounted on carbon-coated copper 75-mesh grids; they were stained with uranyl acetate and lead citrate. A JEOL JEM 1010 electron microscope was used at $80 \mathrm{kV}$, with a TemCam F416 CMOS camera by TVIPS $(4000 \times 4000$ pixels $)$.

\subsection{Behavioral studies}

Rotarod tests were performed at the ages of 7.8, 8.2 and 9 months with an accelerating rotarod apparatus (Ugo Basile). After a training period of two days (first day: $4 \mathrm{rpm}$ for $1 \mathrm{~min}, 4$ repetitions; second day: 4-8 rpm for $2 \mathrm{~min}$, 4 repetitions), on the third day rotarod accelerates from 4 to $40 \mathrm{rpm}$ over $5 \mathrm{~min}$, also with 4 repetitions; the mean latency to fall is calculated. In the retests, only the second day of training is required before the test.

\subsection{Data analysis}

Image quantifications and analyses were carried out with ImageJ/ Fiji. Statistical analyses and charts were done using GraphPad Prism. The level of statistical significance was set to $p<0.05$ in all cases. For the survival analysis, a Kaplan-Meier comparison of the survival curves was carried out (Log-rank Mantel-Cox test). For the rotarod tests, Student's $t$-test was used (mean values with SEM represented). For the distributions of axonal calibers and the proportions of degenerated axons, Fisher's exact test of contingency tables was chosen; error bars represent the $95 \%$ confidence interval, which was calculated following the Wilson/Brown's method.

\section{Results}

As previously described with the PS19 line [15], our mice showed clasping and limb retraction, hunched back, and motor deficits; the paralysis was particularly focalized in the hindlimbs (with a clear muscular atrophy), hence our interest in the integrity of the sciatic nerves. This phenotype worsened at around 10-12 months of age, with a premature death probably due to their inability to feed (Fig. 1a-b). The median survival in the Kaplan-Meier analysis of our transgenic colony was 11.7 months, and the differences between PS19 and Wt littermates survival curves were found to be statistically significant (Fig. 1a). The transgenic mice analyzed on the accelerating rotarod showed a deficit in this motor coordination task that progressively worsened until the difference with Wt performance was statistically significant by the age of 9 months (Fig. 1c).

To test whether this observed hindlimb weakness was associated with P301S tau overexpression in sciatic nerves, we decided to explore by western blot the levels of tau protein in this tissue. Fig. 2a shows the presence of the high-molecular-weight tau isoform "big tau", with an approximate size of $100 \mathrm{kDa}$ and characteristic of the PNS, in Wt and transgenic mice. Tau isoforms without exon $4 \mathrm{~A}$ have a molecular weight of around $52 \mathrm{kDa}$; we observed low levels of endogenous tau in Wt group, while a dramatic increase in PS19 animals, using anti-tau TAU-5 antibody that recognizes both murine and human tau (Fig. 2a). Interestingly, the same increase was found using anti-phospho-tau AT8 antibody, showing that AD-like phosphorylation of transgenic tau is present in the sciatic nerve of PS19 animals (Fig. 2a). To gain insight into which cell populations are overexpressing P301S tau protein, we performed immunohistochemistry with anti-human tau HT7 antibody. In the sciatic nerve of PS19 mice, increased immunoreactivity for P301S tau was present in most of the fibers in transversal and longitudinal 


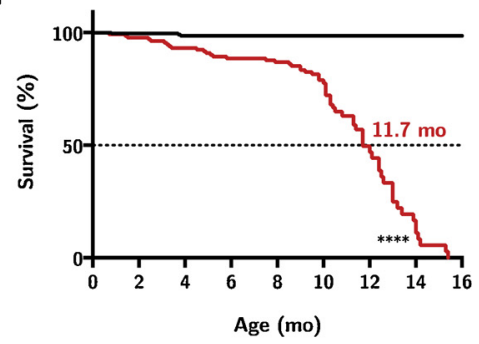

b

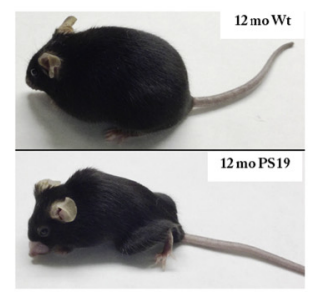

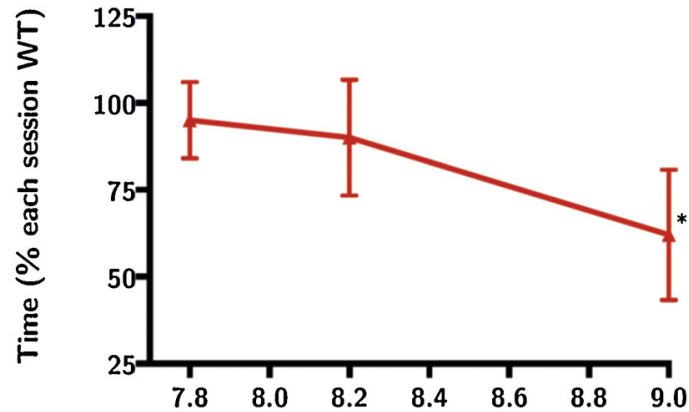

Fig. 1. P301S tau expression progressively impacts on motor function and survival. (a) Kaplan-Meier curves for cumulative survivals show statistically significant differences ( $* * * *$ $p<0.0001$ ) between Wt (black) and PS19 (red) groups; lifespan is shown over a period of 400 days; median survival of PS19 mice is 11.7 months; $n=259 \mathrm{Wt}, 139$ PS19. (b) A representative posture of PS19 mice aged 12 months showing hunched back, muscular atrophy and limb retraction, compared to agematched Wt. (c) Rotarod tests at ages 7.8, 8.2 and 9 months; mean latency to fall from the rod of PS19 mice is shown (percentage of mean Wt time of each test); the difference with Wt time was statistically significant $(* p<0.05)$ at 9 months of age; $n=8-9 \mathrm{Wt}, 6$ PS19. sections (Fig. 2b).

To deep into the ultrastructural changes associated with mutant tau overexpression in the sciatic nerves, we carried out a TEM analysis. Fig. 3a shows the sciatic nerve morphology of Wt and PS19 mice at the age of 9 months. We compared the distributions of the inner diameters of myelinated axons between both groups and no differences were found (Fig. 3b). However, we observed that electron micrographs of the sciatic nerves revealed abnormalities in some myelin sheaths; Fig. 3c shows some examples of three states of increasing degeneration of myelin from Schwann cells (D1-3), by detachment of myelin lamellae at Schmidt-Lanterman clefts, compared to a normal myelin sheath (N). An analysis of these alterations showed a statistically significant increase in the percentage of total degenerating myelinated fibers (D1-3) in PS19 mice when compared to Wt fibers (Fig. 3d).

\section{Discussion}

PS19 mice are a widely used tauopathy model overexpressing human tau protein with the disease-associated P301S mutation driven by Prnp promoter. These mice show important motor deficits that likely result in their death; we showed the worsening of the paralysis with consecutive rotarod tests, and established their median survival at 11.7 months of age. Considering that this paralysis is especially focalized in their hindlimbs, we have investigated the status of their sciatic nerves in order to understand if these motor deficits are due not only to CNS accumulation of pathogenic tau but also to PNS alterations.
Age (mo)

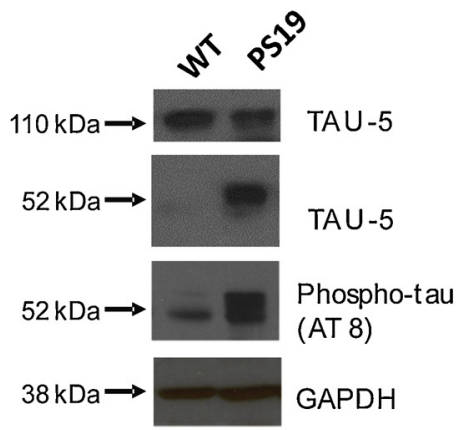

b
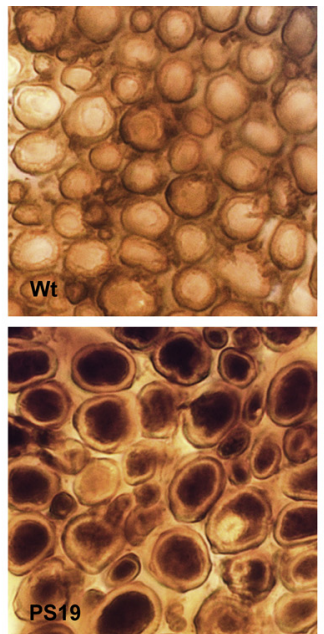

We have observed by western blot and immunohistochemistry that transgenic tau protein is present in the sciatic nerve and phosphorylated in one epitope (Ser202/Thr205, recognized by AT8 antibody) present in different tauopathies as AD [5]. This suggests that some of the phenotypic characteristics of these mice, as are clasping and limb retraction, hunched back and paralysis, can be associated with this expression. The mechanisms underlying the impaired motor behavior are not entirely understood in PS19 mice; it has been suggested that these problems are due to transgene expression in the spinal cord [15]. Our data demonstrate that human pathogenic tau protein driven by Prnp promoter is correlated with an increase in the percentage of degenerating fibers in the sciatic nerves, while the distributions of axonal calibers in Wt and PS19 mice remain similar.

The axonal transport in sciatic nerves is altered in a knockin mice expressing mutant P301 L tau protein [1], as well as in a murine model with human tau carrying mutation K369I [9]. Interestingly, the K369I mouse model is free of degenerative signs in its sciatic nerves. Taking into account our data, it is worthy to question whether the toxic effects of transgenic tau in the PNS are merely due to increased tau levels, to its pathogenic mutation, or because CNS tau (without exon $4 \mathrm{~A}$ ) is more toxic or is able to replace PNS-endogenous big tau (with exon $4 \mathrm{~A}$ ).

Interestingly, similar motor deficits and myelin alterations have been previously reported for tau knockout mice [12], mice with tau lacking exon 10 [7], as well as mice overexpressing tau 0N4R isoform with the same P301S mutation but under the Thy1.2 promoter [14]. Thus, it seems that similar phenotypic alterations can be observed in

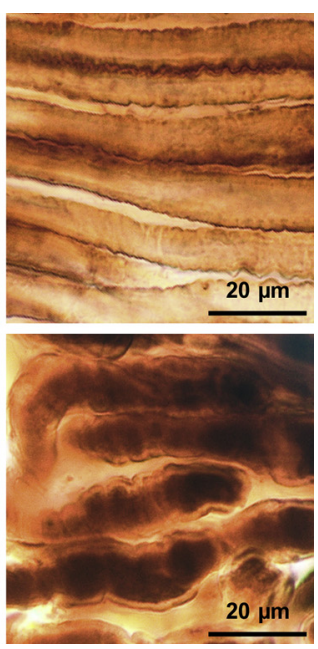

Fig. 2. Overexpression of P301S tau in the sciatic nerve of PS19 mice. (a) Western blot of protein extracts from sciatic nerve of Wt and PS19 transgenic mice probed with the following anti-tau antibodies that recognize both human and murine tau: TAU-5 (total tau) and AT8 (phospho-tau); anti-GAPDH was used as loading control. (b) Immunohistochemistry in transversal (left panels) and longitudinal (right panels) sciatic nerve sections of Wt and PS19 transgenic mice performed with anti-human tau HT7 antibody. Scale bar: $20 \mu \mathrm{m}$. 
a

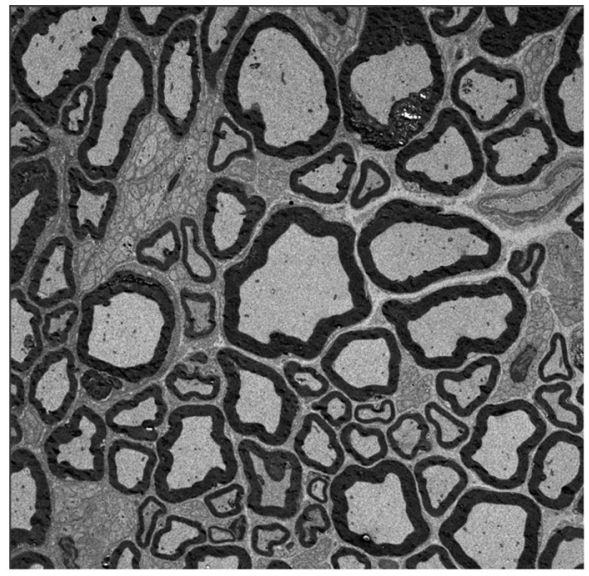

b

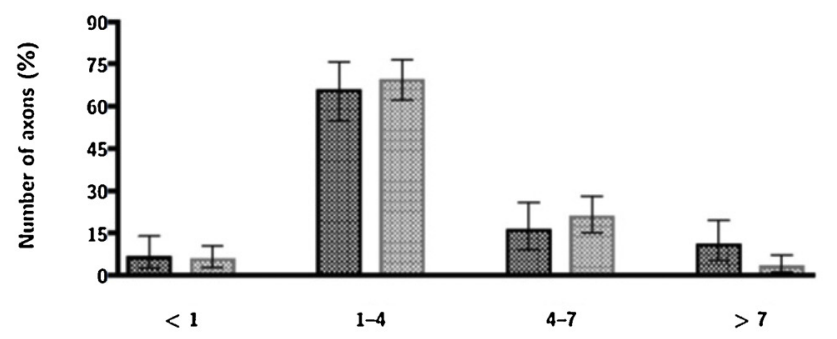

Axon diameter ( $\mu \mathrm{m})$

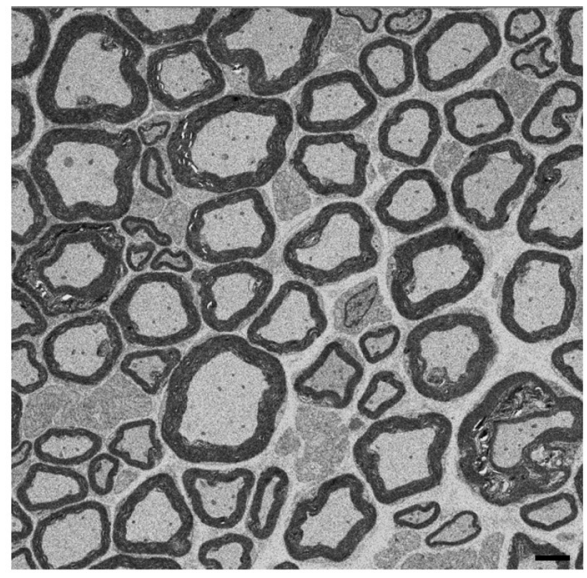

We
Fig. 3. TEM study of the sciatic nerve. (a) Transversal sections of the sciatic nerve of $\mathrm{Wt}$ and PS19 mice at the age of 9 months. (b) Distributions of the inner diameters of myelinated axons in PS19 and Wt mice; no significant differences were found; $n=135 \mathrm{Wt}$, 270 PS19 (axons) [3 Wt, 6 PS19 (mice)]. (c) Images of sciatic nerves showing normal $(\mathrm{N})$ and increasingly degenerated (D1, D2 and D3) myelinated fibers present in PS19 mice. (d) PS19 mice show a statistically significant ( $* * * *$ $p<0.0001$ ) increase in the percentage of degenerating fibers when compared to Wt animals; $n=528 \mathrm{Wt}, 1204$ PS19 (axons) [3 Wt, 6 PS19 (mice)]. Scale bars: (a): $3 \mu \mathrm{m}$, (c): $2 \mu \mathrm{m}$.

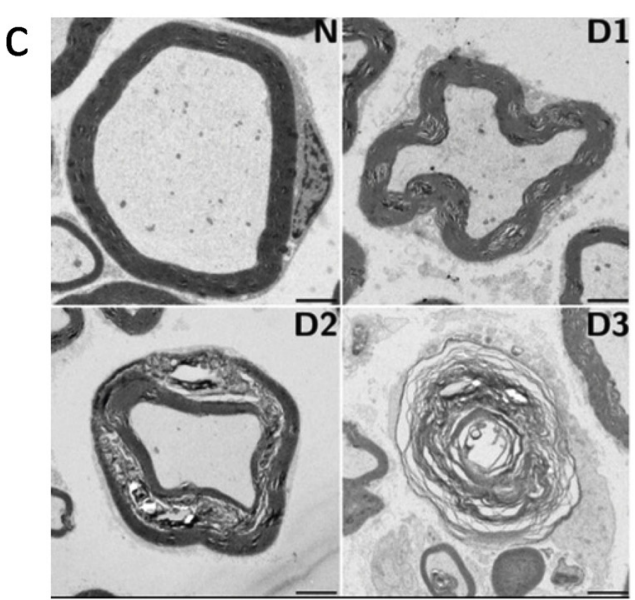

D1

d

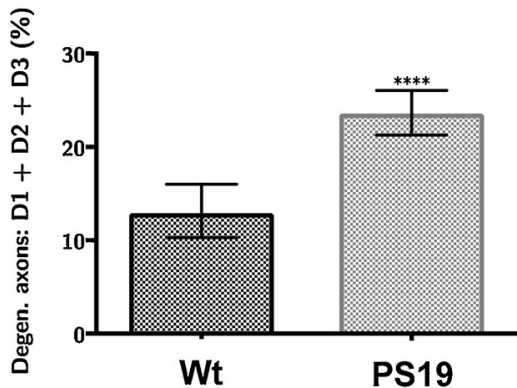

tau animal models with either low or high expression levels, suggesting that an adequate level of tau is necessary in the sciatic nerve; in these studies, an age-related increase in motor deficits is also described. Moreover, an increase in tau protein levels in aged sciatic nerves has been previously reported and related to impaired mechanisms of axonal transport and protein turnover [11].

The paralysis and subsequent death observed in the PS19 model can be due not only to CNS degeneration but also to PNS alterations; in particular, damages to the sciatic nerve may play a crucial role in the observed motor phenotype. Taking into account that the PS19 model is widely used to study tauopathies, we suggest that some characteristics as motor performance or survival afterward drug treatments requires also analyzing the PNS, especially the sciatic nerve. This explanation deserves further testing.

\section{Conflict of interest}

The authors declare no competing financial interests.

\section{Acknowledgements}

This study was funded by grants from Spanish Ministry of Economy and Competitiveness (BFU2016-77885-P), Comunidad de Madrid cofinanced with the Structural Funds of the European Union (S2017/ BMD-3700 (NEUROMETAB-CM)), Centro de Investigación Biomédica en Red sobre Enfermedades Neurodegenerativas (CIBERNED, ISCIII), and from an institutional grant from the Fundación R. Areces. J M-R has a fellowship from Fundación La Caixa.

\section{References}

[1] R. Adalbert, S. Milde, C. Durrant, K. Ando, V. Stygelbout, Z. Yilmaz, S. Gould, J.P. Brion, M.P. Coleman, Interaction between a MAPT variant causing frontotemporal dementia and mutant APP affects axonal transport, Neurobiol. Aging 68 (2018) 68-75.

[2] A. Andreadis, J.A. Broderick, K.S. Kosik, Relative exon affinities and suboptimal splice site signals lead to non-equivalence of two cassette exons, Nucleic Acids Res. 23 (1995) 3585-3593.

[3] U. Bala, K.L. Tan, K.H. Ling, P.S. Cheah, Harvesting the maximum length of sciatic nerve from adult mice: a step-by-step approach, BMC Res. Notes 7 (2014) 714.

[4] D. Couchie, C. Mavilia, I.S. Georgieff, R.K. Liem, M.L. Shelanski, J. Nunez, Primary 
structure of high molecular weight tau present in the peripheral nervous system, Proc. Natl. Acad. Sci. U. S. A. 89 (1992) 4378-4381.

[5] M. Goedert, R. Jakes, E. Vanmechelen, Monoclonal antibody AT8 recognises tau protein phosphorylated at both serine 202 and threonine 205, Neurosci. Lett. 189 (1995) 167-169.

[6] M. Goedert, M.G. Spillantini, R.A. Crowther, Cloning of a big tau microtubule-associated protein characteristic of the peripheral nervous system, Proc. Natl. Acad. Sci. U. S. A. 89 (1992) 1983-1987.

[7] A. Gumucio, L. Lannfelt, L.N. Nilsson, Lack of exon 10 in the murine tau gene results in mild sensorimotor defects with aging, BMC Neurosci. 14 (2013) 148.

[8] K. Iqbal, F. Liu, C.X. Gong, Tau and neurodegenerative disease: the story so far, Nat. Rev. Neurol. 12 (2016) 15-27.

[9] L.M. Ittner, T. Fath, Y.D. Ke, M. Bi, J. van Eersel, K.M. Li, P. Gunning, J. Gotz, Parkinsonism and impaired axonal transport in a mouse model of frontotemporal dementia, Proc. Natl. Acad. Sci. U. S. A. 105 (2008) 15997-16002.

[10] J.L. Jankowsky, H. Zheng, Practical considerations for choosing a mouse model of Alzheimer's disease, Mol. Neurodegener. 12 (2017) 89.

[11] V.S. Krishnan, Z. White, C.D. McMahon, S.I. Hodgetts, M. Fitzgerald, T. Shavlakadze, A.R. Harvey, M.D. Grounds, A neurogenic perspective of sarcopenia: time course study of sciatic nerves from aging mice, J. Neuropathol. Exp. Neurol. 75 (2016) 464-478.

[12] S. Lopes, A. Lopes, V. Pinto, M.R. Guimaraes, V.M. Sardinha, S. Duarte-Silva, S. Pinheiro, J. Pizarro, J.F. Oliveira, N. Sousa, H. Leite-Almeida, I. Sotiropoulos, Absence of tau triggers age-dependent sciatic nerve morphofunctional deficits and motor impairment, Aging Cell 15 (2016) 208-216.

[13] M. Llorens-Martin, C.M. Teixeira, A. Fuster-Matanzo, J. Jurado-Arjona, V. Borrell, E. Soriano, J. Avila, F. Hernandez, Tau isoform with three microtubule binding domains is a marker of new axons generated from the subgranular zone in the hippocampal dentate gyrus: implications for Alzheimer's disease, J. Alzheimers Dis. 29 (2012) 921-930.

[14] Z. Yin, F. Valkenburg, B.E. Hornix, I. Mantingh-Otter, X. Zhou, M. Mari, F. Reggiori, D. Van Dam, B.J.L. Eggen, P.P. De Deyn, E. Boddeke, Progressive motor deficit is mediated by the denervation of neuromuscular junctions and axonal degeneration in transgenic mice expressing mutant (P301S) tau protein, J. Alzheimers Dis. 60 (2017) S41-S57.

[15] Y. Yoshiyama, M. Higuchi, B. Zhang, S.M. Huang, N. Iwata, T.C. Saido, J. Maeda, T. Suhara, J.Q. Trojanowski, V.M. Lee, Synapse loss and microglial activation precede tangles in a P301S tauopathy mouse model, Neuron 53 (2007) 337-351. 